\title{
Pyramidal signs in a Caucasian patient with spinal muscular atrophy: a case report
}

\author{
Yu Wan ${ }^{1}$, Jun Zhang ${ }^{2}$ \\ ${ }^{1}$ Department of Neurology, Qingdao Municipal Hospital, Shandong, ²Department of Neurology, Peking University People's Hospital, \\ Beijing, China
}

\begin{abstract}
Spinal muscular atrophy (SMA), an autosomal recessive disease, is characterized by the selective loss of spinal motor neurons due to reduced levels of the survival motor neuron (SMN) protein. The clinical symptoms of SMA are progressive proximal muscle weakness and paralysis. Here we describe a 20-year-old Turkmenistan male with SMA who presented with uncommon pathological reflexes and asymmetric onset of weakness. The diagnosis after genetic analysis revealed a homozygous deletion of SMN1 exons seven and eight. The copies of SMN2 exon seven were normal. Although pyramidal signs are not a common symptom of SMA, they could not be used to exclude the diagnosis of SMA in a patient with neuromuscular degenerative symptoms. Therefore, an additional attention is warranted to SMA patients with pathological reflexes.
\end{abstract}

Key words: spinal muscular atrophy, pyramidal sign, amyotrophic lateral sclerosis.

\section{Introduction}

Spinal muscular atrophy (SMA) is an autosomal recessive disease characterized by the selective loss of spinal motor neurons. This loss is due to reduced levels of the survival motor neuron (SMN) protein [10]. Spinal muscular atrophy is the leading genetic cause of infant mortality, and there are currently no effective treatments to slow progression of this disease $[4,9]$. Humans have two genes that produce SMN, SMN1 and SMN2, the former of which is deleted or nonfunctional in the majority of patients with SMA. These two genes are nearly identical with one exception being a cytosine to thymine transition within exon seven of SMN2. This transition induces the exclusion of exon seven from $90 \%$ of the SMN2 mRNA transcript. The truncated
SMN protein is then rapidly degraded [7]. Consequently, most of SMN2 mRNA transcripts are not able to compensate for the loss of SMN1 in SMA patients. Thus, the SMN2 copy number, proportional to the intact mRNA transcripts, is considered to be a modifying factor for the clinical severity of SMA [11]. The clinical symptoms of SMA are progressive proximal muscle weakness and paralysis. The differential diagnostic conditions include polymyositis and myodystrophy. Spinal muscular atrophy is clinically classified into four phenotypes based on the age of onset and motor function achieved. Spinal muscular atrophy type I has an onset of clinical signs before six months of age, type II between seven and eighteen months, type III between eighteen 
months and eighteen years, and type IV patients have adult onset [1].

\section{Case report}

A 20-year-old Turkmenistan male was hospitalized in March 2013 complaining of progressive limb weakness that he had experienced for the previous eight years. In otherwise good health, the patient suffered from weakening of the right lower limb muscles, which caused him to occasionally stumble, since 2005. In 2007, his left lower limb presented with similar symptoms that were at times accompanied by myoclonic jerks. The weakness in both lower limb muscles became progressively worse, which led to difficulties in walking and an inability to run and jump. Then in 2011, the patient developed weakening of both upper limb muscles, which did not affect his ability to write or move his upper limbs. The patient did not experience dysphagia or choking while drinking. The patient also had no family history of neuromuscular disease.

Upon physical examination, the patient was attentive and coherent and demonstrated normal hearing and vision. No atrophy or fasciculation of the tongue or facial weakness were observed. The patient exhibited normal strength of the sternocleidomastoid and cervical muscles and moderate muscular tension in his limbs. Neurological examination revealed muscle atrophy involving both the upper and lower extremities. The weakness was symmetrical and more proximal than distal, with the lower limbs generally being weaker than the upper limbs. A winged scapula and a pes cavus were observed. The patient exhibited a bilateral biceps strength of grade 5-, a triceps strength of grade 4, a bilateral iliopsoas strength of grade 2 , and a grade 5 - strength of the quadriceps, bilateral tibialis anterior and gastrocnemius. The grip strength of his hands was grade 5-. He had a Gower's sign and a waddling gait. Sensory function tests revealed no abnormalities. Deep tendon reflexes were tested and displayed a bilateral biceps reflex (+/++). The bilateral triceps reflex, Achilles tendon reflex and patellar tendon reflex were not elicited. The patient also had a bilateral Babinski's sign (Fig. 1) and Chaddock's sign.

Auxiliary examination showed the patient's creatine kinase (CK) levels to be $972 \mathrm{U} / \mathrm{l}$, CK isoenzyme (CK-MB) levels to be $38 \mathrm{U} / \mathrm{l}$, and lumbar pressure to be $224 \mathrm{~mm} \mathrm{H}_{2} \mathrm{O}$. Routine cerebrospinal fluid tests, electrocardiogram (ECG), ultrasonic cardiogram and magnetic resonance imaging (MRI) examinations were unremarkable. Electromyography (EMG) indicated nerve damage on the bilateral first interosseous muscle, bilateral tibialis anterior, right quadriceps and bilateral rectus abdominis. A prolonged latent period was found in the cortex section of the deep sensory paths in the bilateral lower limbs. Both the lower limb motor evoked potentials had a decreased amplitude in the $L 4$ nerve root. Muscle biopsy of the right biceps revealed angular atrophic fibers, and a nicotinamide adenine dinucleotide tetrazolium reductase (NADH-TR) stain showed large fiber-type groups, demonstrating muscular "neurogenic" damage (Fig. 2). A multiplex ligation-dependent probe amplification (MLPA) kit using P021 probes was utilized to detect deletion and/or duplication of the SMN1/SMN2 gene (Microbiology Research Centre, Holland, Amsterdam, The Netherlands). This examination discovered a homozygous deletion of SMN1 exons seven and eight. The relative peak height ratios of SMN2 exon eight increased to approximately 1.5 , indicating that three copies of this exon were present. SMN2 exon 7 was present at the normal copy number of two (Fig. 3). The patient was therefore diagnosed as having SMA type III based on the age of onset, disease progression and genetic test results.

\section{Discussion}

Spinal muscular atrophy is a severe neuromuscular disease that primarily involves the lower motor neurons in the spinal cord and brainstem, resulting in progressive muscular atrophy, fasciculation and decreased tendon reflex. The patient described here had the basic characteristics of SMA, however, he

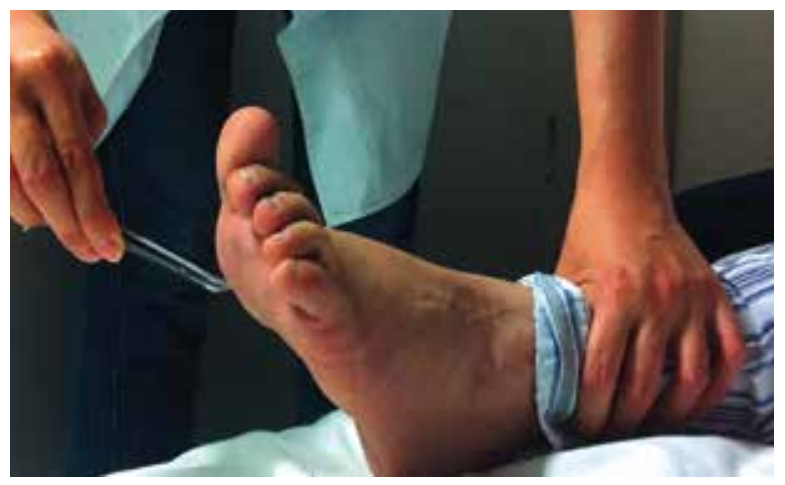

Fig. 1. Evidence of the Babinski's sign. 

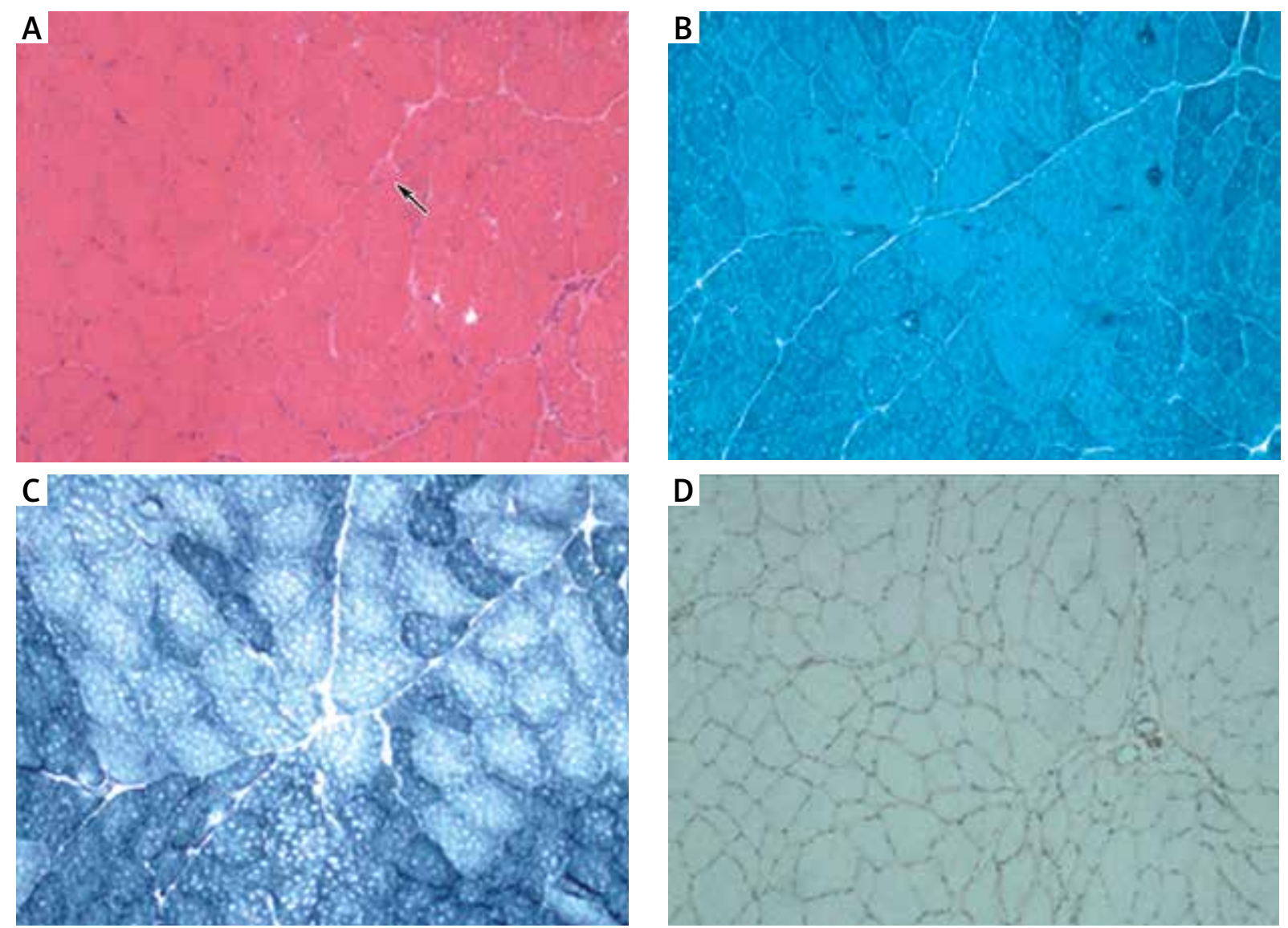

Fig. 2. Muscle biopsy of the right bicep (× 10). A) Hematoxylin and eosin staining reveals angular atrophic fibers (arrow). B) Gomori's trichrome staining indicates that there is no accumulation of abnormal materials. C) NADH-TR staining shows large fiber-type groups. D) The muscle positive stain dysferlin.

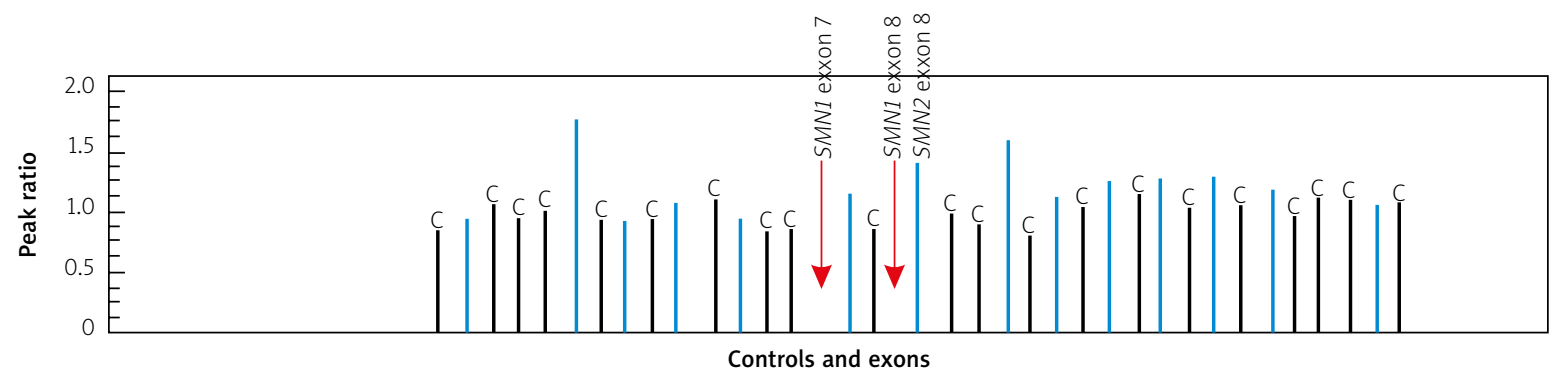

Fig. 3. Copy number analysis of SMN1 and SMN2. The red arrows indicate homozygous deletions of SMN1 exons seven and eight. The relative peak height ratio of SMN2 exon eight increases to approximately 1.5, indicating the presence of three copies.

presented with pathological reflexes, which indicated an involvement of the pyramidal tract. There were no other etiologies to explain this rare condition. We had considered juvenile amyotrophic lateral sclerosis (ALS) during the procedure of diagnosis because the pathological reflex frequently indicates ALS. Amyotrophic lateral sclerosis, the most common and best-recognized form of motor neuron diseases, is characterized by a relentless degeneration of both upper and lower motor neurons leading to 
progressive muscular paralysis [5]. Mutations in the superoxide dismutase (SOD1) and fused in sarcoma (FUS) genes are responsible for approximately $20 \%$ and $4-6 \%$ of familial ALS, respectively [12]. In the case presented here though, genetic tests confirmed the diagnosis of SMA-III and not ALS.

It is currently unknown whether SMA and ALS share molecular pathways in the process of motor neuron degeneration. As is common with neuromuscular degenerative disorders, the pathogenesis of ALS remains unclear. The multiple mechanisms thought to play a role in this disease include genetic factors, oxidative stress, excitotoxicity and protein aggregation, as well as impairments in RNA processing, axonal transport and mitochondrial function [2]. Synapses and distal axonal compartments are frequently involved in the early stages of ALS [6]. Mutations in FUS induce a redistribution of SMN from the axon to cytosolic FUS accumulations, which disrupt the function of SMN, thus leading to axonal defects [3]. Moreover, a study of 600 sporadic ALS patients found that an abnormal number of SMN 1 copies (one or three rather than two) occurred more frequently in cases than controls $(\mathrm{OR}=2.8,95 \% \mathrm{Cl}: 1.8-4.4)$ [8]. Since the SMN1 protein is ultimately affected in both ALS and SMA, a shared pathogenesis of these two disorders is very likely. Additional studies examining the molecular mechanisms involved in these two conditions should be performed.

No reports concerning pyramidal tract damage in Asian SMA patients exist. Symptoms of SMA in patients of various ethnicities should be further explored and compared to determine whether there are significant differences. In addition, patients with SMA typically present with symmetrical onset. One of the noteworthy characteristics of this case is that the patient's left lower limb experienced weakness two years after the right lower limb. This patient should be followed closely so that the differences in disease progression between this unique case of SMA and classical cases can be compared.

In conclusion, pyramidal signs cannot be used to exclude the diagnosis of SMA in patients with neuromuscular degenerative symptoms. While the cause of the SMA remains unclear, further investigation of SMA patients with pathological reflexes is necessary.

\section{References}

1. D’Amico A, Mercuri E, Tiziano FD, Bertini E. Spinal muscular atrophy. Orphanet J Rare Dis 2011; 6: 71.

2. Ferraiuolo L, Kirby J, Grierson AJ, Sendtner M, Shaw PJ. Molecular pathways of motor neuron injury in amyotrophic lateral sclerosis. Nat Rev Neurol 2011; 7: 616-630.

3. Groen EJ, Fumoto K, Blokhuis AM, Engelen-Lee J, Zhou Y, van den Heuvel DM, Koppers M, van Diggelen F, van Heest J, Demmers JA, Kirby J, Shaw PJ, Aronica E, Spliet WG, Veldink JH, van den Berg LH, Pasterkamp RJ. ALS-associated mutations in FUS disrupt the axonal distribution and function of SMN. Hum Mol Genet 2013; 22: 3690-3704.

4. Kayadjanian N, Burghes A, Finkel RS, Mercuri E, Rouault F, Schwersenz I, Talbot K. SMA-EUROPE workshop report: Opportunities and challenges in developing clinical trials for spinal muscular atrophy in Europe. Orphanet J Rare Dis 2013; 8: 44.

5. Luigetti M, Lattante S, Conte A, Romano A, Zollino M, Marangi G, Sabatelli M. A novel compound heterozygous ALS2 mutation in two Italian siblings with juvenile amyotrophic lateral sclerosis. Amyotroph Lateral Scler Frontotemporal Degener 2013; 14: 470-472.

6. Murray LM, Talbot K, Gillingwater TH. Review: neuromuscular synaptic vulnerability in motor neurone disease: amyotrophic lateral sclerosis and spinal muscular atrophy. Neuropathol Appl Neurobiol 2010; 36: 133-156.

7. Porensky PN, Burghes AH. Antisense oligonucleotides for the treatment of spinal muscular atrophy. Hum Gene Ther 2013; 24: 489-498.

8. Schymick JC, Talbot K, Traynor BJ. Genetics of sporadic amyotrophic lateral sclerosis. Hum Mol Genet 2007; 16 Spec No. 2: R233-242.

9. Sen A, Dimlich DN, Guruharsha KG, Kankel MW, Hori K, Yokokura T, Brachat S, Richardson D, Loureiro J, Sivasankaran R, Curtis D, Davidow LS, Rubin LL, Hart AC, Van Vactor D, Artavanis-Tsakonas S. Genetic circuitry of Survival motor neuron, the gene underlying spinal muscular atrophy. Proc Natl Acad Sci U S A 2013; 110: E2371-2380.

10. Sleigh JN, Barreiro-lglesias A, Oliver PL, Biba A, Becker T, Davies KE, Becker CG, Talbot K. Chondrolectin affects cell survival and neuronal outgrowth in in vitro and in vivo models of spinal muscular atrophy. Hum Mol Genet 2014; 23: 855-869.

11. Zheleznyakova GY, Kiselev AV, Vakharlovsky VG, Rask-Andersen M, Chavan R, Egorova AA, Schioth HB, Baranov VS. Genetic and expression studies of SMN2 gene in Russian patients with spinal muscular atrophy type II and III. BMC Med Genet 2011; 12: 96.

12. Zou ZY, Cui LY, Sun Q, Li XG, Liu MS, Xu Y, Zhou Y, Yang XZ. De novo FUS gene mutations are associated with juvenileonset sporadic amyotrophic lateral sclerosis in China. Neurobiol Aging 2013; 34: 1312.e1-8.

\section{Disclosure}

Authors report no conflict of interest. 